\title{
Un análisis del proyecto "Mejora del acceso de niños, niñas y adolescentes a los servicios de traumatología ortopédica en el sur de la Franja de Gaza" desde la visión de un traumatólogo (Intervención con Equipos Quirúrgicos Especializados, EQE)
}

\author{
Felipe Noya Álvarez \\ Cirujano Ortopédico y Traumatólogo Hospital Clínico Universitario de Santiago de Compostela, Unidad de \\ Ortopedia Infantil. Voluntario de Médicos del Mundo; felipe.noya@medicosdelmundo.org; ORCID id: \\ https://orcid.org/0000-0002-0099-8187
}

DOI: https://doi.org/10.37536/RIECS.2020.5.S1.181

Recibido: 04/02/2020; Aceptado: 05/02/2018; Publicado: 14/02/2020

Resumen: Se resume la actividad efectuada por el autor, traumatólogo miembro de un Equipo Quirúrgico Especializado (EQE) en un proyecto de Cirugía Ortopédica Infantil en hospitales de la Franja de Gaza. La organización Médicos del Mundo (MdM) es la que implementa el proyecto desde 2015 hasta hoy con varias misiones quirúrgicas. La situación de crisis y conflicto político mantenido en el tiempo en la zona, con estallidos violencia militar repetidos, hacen compleja la implementación del programa. Se pretende hacer una descripción del programa, del modo de trabajo de los EQE de cada misión, y resaltar las dificultades detectadas por los profesionales. Se enumeran los resultados de la actividad efectuada en cuanto a la labor asistencial con los pacientes y de formación y capacitación con los colegas locales. No se pueden entender las dificultades de implementación del proyecto si no se conoce el contexto del bloqueo a las personas y materiales que sufre la Franja, la debilidad de sus estructuras políticas, duplicadas en algunos casos, así como el contexto socioeconómico. Incluso se muestra este ámbito de la patología como poco dimensionada en importancia en términos de salud pública de una población. Las dificultades de movilidad de los profesionales de la Franja de Gaza hacen que tengan casi imposible la actualización y capacitación para el tratamiento de casos complejos. La falta de datos epidemiológicos fiables no permite organizar una respuesta asistencial ad hoc. La multiplicidad de actores en el ámbito asistencial tampoco ayuda a dar una respuesta organizada.

Palabras Clave: Ortopedia Infantil, Franja de Gaza, Equipos Quirúrgicos Especializados, Displasia de Cadera, Pie Zambo, Placas de Osteotomía, Misión, Consultas Externas, Formación durante la tarea.

\begin{abstract}
This article summarizes the activity carried out by a orthopaedic surgeon, the author, member of Specialized Surgical Team (SST) in a Project of Orthopaedic Child's Surgery in GazaHospitals. The organization Doctors of the World (MdM) is the one that implements the project from 2015 to today with several surgical missions. The situation of crisis and political conflict maintained over time in the area, with repeated outbreaks of military violence make theimplementation of the program complex. It is intended in the article to make a description of the program, the way of working the SQE of each mission, and highlight the difficulties detected by professionals. The results of the activity carried out in terms of patient care and training and trainingof local colleagues are listed. The difficulties of implementing the project cannot be understood if the context of blocking people, and materials suffered by the strip, the weakness of its duplicate political structures in some cases as well as the socio-economic context is not known. This field of pathology is even shown as undersized in terms of public health and the future of a
\end{abstract}


population. The mobility difficulties of Gaza Strip professionals make it almost impossible to update and train complex cases. Lack of reliable epidemiological data does not allow an ad hoc care response to be organized. The multiplicity of actors in the field of care also does not help an organized response.

Key words: Children's Orthopaedic Surgery, Gaza Strip, Specialized Surgical Teams, Hip developmental dysplasia, Clubfoot, Osteotomy plates, Mission, External Consultations, Training in working.

\section{Introducción}

La historia de Palestina de los últimos años está marcada por el conflicto con Israel que podría iniciarse en el año 1948, fecha del fin del Protectorado Británico y de la Primera Guerra árabe-israelí. En junio de 1967, durante la Guerra de los Seis Días, Israel ocupó Gaza ciudad y un territorio de casi $50 \mathrm{~km}$ de largo y apenas $10 \mathrm{~km}$ de ancho, la llamada Franja de Gaza, anteriormente bajo ocupación egipcia. Con el traspaso gradual del control a la Autoridad Palestina de algunos territorios (TOP), fruto de los Acuerdos de Oslo en 1994 [1], la Franja es abandonada paulatinamente por Israel, pero el estancamiento de dichas negociaciones supone reagudizaciones sucesivas del conflicto. Israel decide la construcción de un "muro defensivo" no sólo en Gaza sino también en el resto de TOP, e inicia el establecimiento de numerosos puntos de control, checkpoints, que en el caso de Gaza se convierte en una auténtica frontera con restricción de paso de personas y materiales [2].

La presión política, no sólo de Israel sino también de parte de la comunidad internacional, obliga a Egipto al cierre del paso desde el sur de Gaza, a través de la localidad de Rafah, y se convierte esta ruta en insegura, debido a los muchos cierres y a la inseguridad de todo tipo en la Península del Sinaí. Más de 2000 personas en Gaza han cursado solicitudes a las autoridades israelíes para cruzar el control fronterizo por Erez, norte de la Franja, y recibir asistencia sanitaria en el algún lugar del territorio palestino o en Jerusalén Este. Muchas serán denegadas o retrasadas sin respuesta [3]. Aproximadamente dos millones de personas viven en la Franja de Gaza, $365 \mathrm{~km}^{2} \mathrm{de}$ superficie lo que supone una densidad de 4167 hab./ $\mathrm{km}^{2}$ [4]. Según Naciones Unidas (ONU) a través de la Agencia de Naciones Unidas para los Refugiados de Palestina en Oriente Próximo (UNRWA), y de la Oficina de Naciones Unidas para la Acción Humanitaria (OCHA), hasta 1,3 millones de gazatíes requieren ayuda humanitaria, el suministro de electricidad sólo cubre el $20 \%$ de las necesidades, incluso los hospitales, el $97 \%$ del agua corriente no es potable, y el $47 \%$ de los hogares no tienen seguridad alimentaria $[5,6]$.

Médicos del Mundo-España (MdM), así como también su Red Internacional está presente en los Territorios Ocupados Palestinos (TPO) desde el 2004 y trabajando en Jordania trabajando en campos con refugiados palestinos desde 1990. En los últimos años está presente en TPO, tanto en la Franja de Gaza como en Cisjordania, con múltiples proyectos de salud pública, comunitaria, equipos quirúrgicos especializados (EQE) [7], salud mental.

Como ejemplo de salud mental: "Protección y Apoyo Psicosocial a la población afectada o en riesgo de violaciones del Derecho Internacional Humanitario, con especial énfasis a los grupos vulnerables, mujeres, menores de edad y población beduina de las Gobernaciones de Jericó y Tubas (Área C) y la periferia de Jerusalén Este (E1), Territorio Palestino Ocupado".

Como ejemplo de trabajo con EQE varias iniciativa pasadas como en respuesta a los ataques militares en Gaza “Operación Lluvia de Verano" en el año 2006, el "Programa de apoyo a personal médico y paramédico del sistema de salud en los hospitales Nasser, Kamal Aduan y Shifa, refuerzo capacidades asistenciales en traumatología a pacientes víctimas de los bombardeos", que se implementó con el apoyo de la Sociedad Española de Medicina de Urgencias (SEMERGEN), replicado en el año 2008 durante la "Operación Plomo Fundido". Otro ejemplo de trabajo con EQE, implementado por MdM y todavía en activo, es un proyecto desarrollado bajo la coordinación e iniciativa de ECHO/OCHA "Projects of surgeries trauma lortho / LR plastic" para realizar cirugía 
reconstructiva compleja de paciente heridos durante el conflicto en la valla fronteriza de Gaza por las conmemoraciones de la Nakba, o retorno a su tierra de los refugiados palestinos que comenzaron en marzo de 2018 y se mantienen en la actualidad [8-11].

El objeto del presente artículo está centrado en el trabajo del autor como Traumatólogo en las sucesivas misiones, que, desde enero de 2015, meses después de los bombardeos del verano de 2014 "Operación Margen Protector", hasta la actualidad, a falta de dos meses para una nueva misión, dentro del "Proyecto de Mejora del acceso de niños, niñas y adolescentes a los servicios de traumatología ortopédica en el sur de la Franja de Gaza". Se trata de un proyecto basado en EQE, desarrollado durante ya más de cinco años. Los equipos ayudan en el tratamiento de enfermedades y deformidades del aparato locomotor de niños y niñas. Es importante reseñar que el diseño del presente artículo se hace desde un enfoque referido a la actividad de un traumatólogo. Por tanto, aquellas conclusiones o discusiones que el resto de las aproximaciones que se puedan hacer, por ejemplo, anestesia, enfermería, salud pública, incidencia política, no están contempladas más que de modo colateral, y no se ha entrado a valorar posibles contradicciones o coincidencias en el mismo.

De la actividad realizada durante todo este tiempo de funcionamiento del proyecto, hemos podido extraer ya muchas lecciones aprendidas, con el fin de hacer una mejor y más adaptada intervención en relación a los objetivos esperados, que en realidad se han ido modificando también. En todo este tiempo, la formulación sigue teniendo la constante de la actividad basada en EQE, y es objeto de este artículo analizar las capacidades de este formato de intervención. Sin duda la influencia del contexto sociopolítico en la Franja, el bloqueo fronterizo a personas y materiales, la capacidad de la organización MdM de trasladar a voluntariado hasta el terreno, la división interna entre Gobierno de facto, Hamás y la Autoridad Palestina, la debilidad de las infraestructuras, son elementos claves que influyeron en la implementación del proyecto y la realización de varias adaptaciones.

Nos hacemos varias preguntas: ¿Es un programa o proyecto con EQE un método con impacto significativo sobre la salud? ¿Podemos proponer algún cambio en el proyecto para la mejora de la calidad del mismo? ¿Detectamos otro valor añadido al proyecto además de aquellos objetivos escritos en su formulación? ¿En un contexto tan complejo, es posible alguna acción que desde el proyecto proponga alguna alternativa que lo mejore?

Los datos del artículo se han extraído a partir de las formulaciones e informes de actividad del proyecto citado. Este proyecto ha sido implementado a través de varias misiones con EQE, desde 2015 hasta hoy día. Las tablas de resultados se presentan en inglés, dado que es así como se presentan en los informes, si bien se trata de tecnicismos que pueden ser comprendidos por los lectores con formación científica.

Se diseñó un formulario para cada paciente donde se recogen los principales ítems, (Tabla I). En la misma se pretende incluir no sólo el diagnóstico y tratamiento efectuado sino también otras opciones terapéuticas y datos epidemiológicos someros. Se pretende también hacer coincidir y correlacionar con marcadores numéricos propios del hospital con la finalidad de poder dar continuidad asistencial al paciente para ulteriores revisiones por el EQE o por los traumatólogos locales.

Tabla I. Formulario para pacientes. Fuente. Elaboración propia a partir de Médicos del Mundo España

\begin{tabular}{|l|l|l|l|l|l|}
\hline Name: & & $\begin{array}{l}\text { Gender: } \\
\text { M or F }\end{array}$ & Age: & & ID \#: \\
\hline Hospital File \#: & & Contact \#: & & & Surgeon: \\
& & & & \\
\hline
\end{tabular}




\begin{tabular}{|c|c|c|c|c|}
\hline Diagnosis: & & & & \\
\hline Operation: & & & & \\
\hline $\begin{array}{l}\text { Dressing } \\
\text { Recommendatio } \\
\mathbf{n}\end{array}$ & Date of first dressing: & & $\begin{array}{l}\text { Frequenc } \\
\text { y of } \\
\text { dressing: }\end{array}$ & \\
\hline & Type of wound: & & & \\
\hline & Type of dressing: & & & \\
\hline Microbiology: & $\begin{array}{l}\text { Conducted: } \\
\text { Yes or No }\end{array}$ & & Result: & \\
\hline & Antibiotic Sensitivity: & & $\begin{array}{l}\text { Treatmen } \\
\mathbf{t} \\
\text { Duration: }\end{array}$ & \\
\hline $\begin{array}{l}\text { Physiotherapy } \\
\text { Recommendatio } \\
\mathrm{n}:\end{array}$ & $\begin{array}{l}\text { Physiotherapy: } \\
\text { Yes or No }\end{array}$ & & $\begin{array}{l}\text { Weight } \\
\text { Bearing: } \\
\text { No } \\
\text { Partial } \\
\text { Full }\end{array}$ & \\
\hline & $\begin{array}{l}\text { Request for specific } \\
\text { treatment: }\end{array}$ & & & \\
\hline $\begin{array}{l}\text { Surgeon's } \\
\text { Comments: }\end{array}$ & & & & \\
\hline $\begin{array}{l}\text { Surgeon's } \\
\text { Signature: }\end{array}$ & & Date: & & \\
\hline
\end{tabular}

El inicio del mismo se formula a partir de una primera misión realizada por el autor en enero de 2015. Esta misión tenía un margen temporal de 15 días, y estaba localizada no sólo en el Sur de la Franja, Hospital Nasser de Khan Younis, sino también en Gaza City, Hospital Al-Shifa. Debo reseñar que se trata de los dos centros hospitalarios más grandes de la Franja. El carácter de la misión estaba basado en una exploratoria por parte de la organización MdM, que responde a una solicitud por parte de las autoridades sanitarias del ministerio de Salud $(\mathrm{MoH})$, muy satisfechas por el valor añadido que el "Programa de Refuerzo de Capacidades del personal médico para atención de pacientes de traumatología". Durante esa misión sólo se movilizó el recurso de un traumatólogo especializado en Ortopedia Infantil, el autor, que visitó esos dos hospitales y sus Servicios de Traumatología. Además de la actividad asistencial en ambas durante ocho días de trabajo asistencial, dos jornadas de consulta externa ambulatoria con 130 pacientes consultados, y seis jornadas de actividad quirúrgica, se efectuó una jornada con un evento al que se invitó a personal sanitario con responsabilidades en Ortopedia Infantil, de la totalidad de la Franja, pediatras, enfermería, epidemiólogos, para debatir al respecto de la idoneidad de un proyecto de refuerzo de capacidades en la materia. 
En las sucesivas misiones el proyecto se realizó en ambos los dos hospitales citados, además del European Hospital de Khan Younis. El carácter de mejora de las capacidades del proyecto hizo que la mayoría de las últimas misiones se centrase en el sur de la Franja, como se formula en la convocatoria y es por ello que fue en el Hospital Nasser dónde se efectuaron la mayoría de las misiones.

Estamos analizando pues un proyecto basado distintas misiones con EQE con un desarrollo temporal desde enero 2015 hasta una misión proyectada para el próximo marzo 2020. La necesidad de dar seguimiento clínico y de resultados está en íntima relación con la patología que nos ocupa. El tratamiento de lesiones y/o malformaciones del aparato locomotor de los niños y niñas que aún está en crecimiento y en muchos casos precisan más de una intervención quirúrgica. En las reformulaciones del proyecto se puso énfasis a esto y se decidió incluir en lo posible en consultas externas la posibilidad mayor de incluir en la agenda a pacientes ya tratados en misiones anteriores. Ello obligó también a procurar alguna repetición en los miembros del equipo, así como a centrar la intervención en un único hospital. Es importante reseñar las dificultades que las familias de muchos pacientes tenían para poder desplazarse dentro de la Franja.

Cada uno de los EQE, después de la primera misión, estuvieron formados por cuatro personas (dos traumatólogos con experiencia en infantil, un anestesista con experiencia en infantil y una enfermera instrumentista). El material quirúrgico, y equipamiento utilizado en las misiones fue el disponible en los hospitales. En general se dispone del uso de un quirófano del bloque durante cada una de las jornadas, que suelen ser cinco por misión, en horario de jornada prolongada, habitualmente de ocho de la mañana a seis de la tarde, con una parada de descanso comida en la actividad quirúrgica. El personal del centro apoya al equipo quirúrgico durante toda la actividad. Es especialmente reseñable, el apoyo de traumatólogos del hospital interesados y con responsabilidades sobre los pacientes, pues la continuidad asistencial de los pacientes será suya. Además, la actividad de la persona de enfermería supervisora del área quirúrgica y personal de enfermería fue muy importante. Quisiera resaltar aquí las actividades de formación realizadas en el ámbito de la profilaxis de la infección quirúrgica, por la enfermera del EQE, que acudió con el mismo en múltiples misiones. Como he indicado previamente me voy a centrar en el artículo en la actividad del ámbito traumatológico, quizá en un futuro se podría completar con un análisis más global del proyecto.

Las aprobaciones y permisos de entrada y salida por parte de Israel, de la autoridad palestina, como también del gobierno de facto en la Franja, Hamás, (Figura 1) supone un proceso burocrático lento. Las coordinaciones o FN son números que identifican ante las autoridades fronterizas israelíes a las personas para entrar por el paso de Erez (Figura 2), único posible de entrada en la Franja. El proceso de verificación de todos estos permisos, tanto para la entrada como la salida, suponen un tiempo de entre una a tres horas. En ese checkpoint también se vigilan los materiales e instrumentos que se transportan. En ocasiones el material médico, aunque sea debidamente consignado previamente, podría ser inmovilizado, o incluso decomisado.

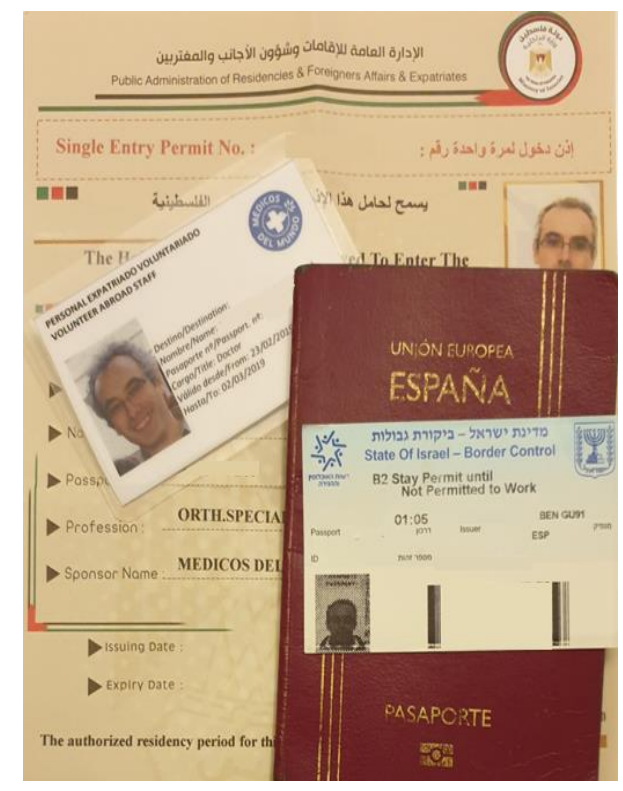

Figura 1 Permisos y aprobaciones del autor para la entrada y salida de la Franja 


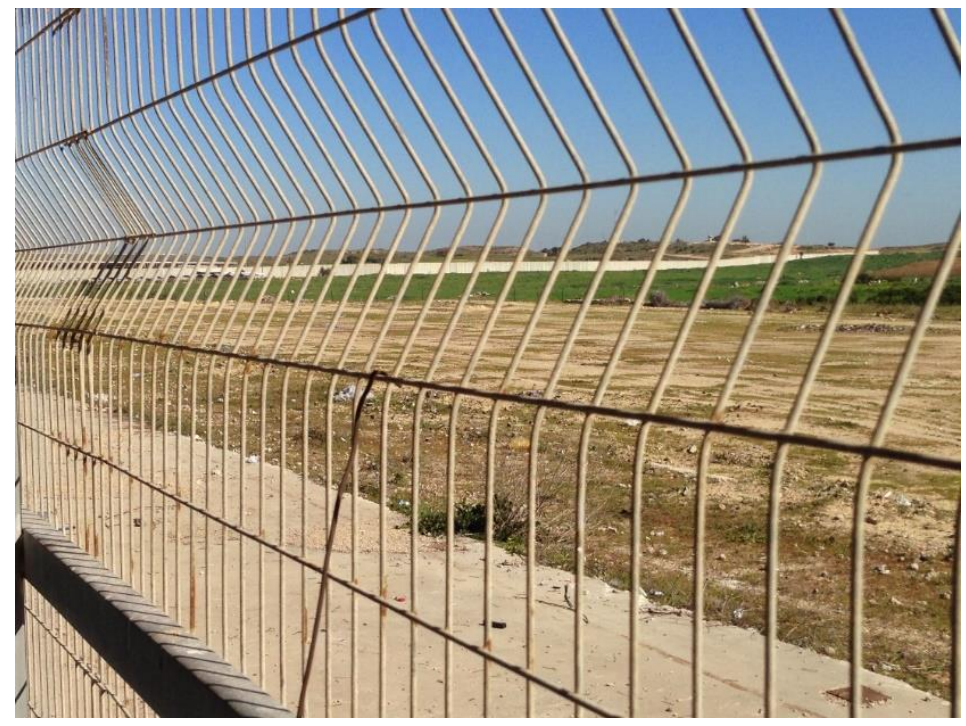

Figura 2 Paso de Erez

La actividad quirúrgica en el ámbito de la ortopedia infantil, comparada con otras partes de la Traumatología tiene una demanda menor de material quirúrgico caro o complejo. Sin embargo, hay importantes excepciones, como es el caso de placas de osteotomías, fijadores externos para correcciones y alargamientos óseos, cirugía mínimamente invasiva, enclavados óseos con sistemas que preserven las zonas de crecimiento de los huesos, etc. (Figura 3). En muchas de ellas no hay opciones o alternativas válidas para compensar la ausencia de dicho material.

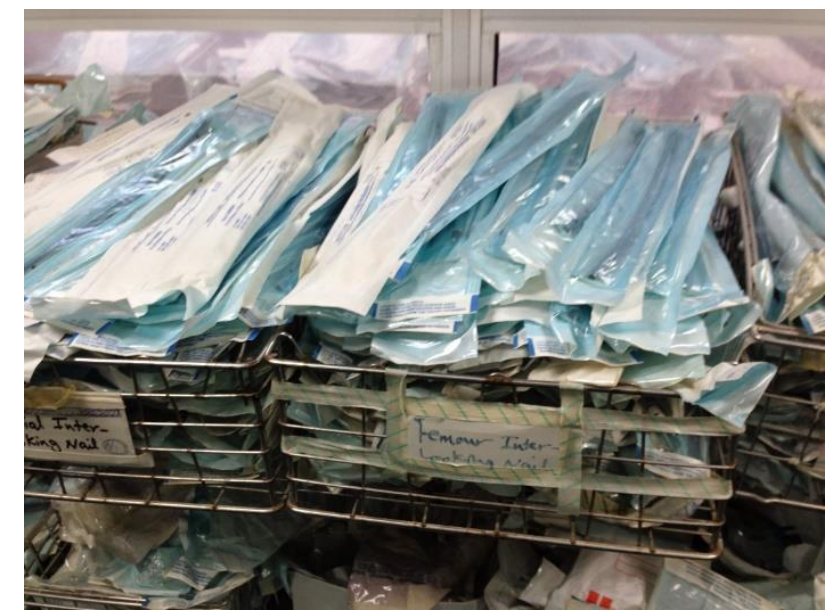

Figura 3 Material quirúrgico.

A la dificultad para el transporte y envío del material se suma también el recorte de la ayuda humanitaria en España y el que la salud haya dejado de ser un sector prioritario para España en la zona. Los distintos EQE han tenido que trabajar y seleccionar sus pacientes teniendo en cuenta la realidad de los quirófanos de la Franja. Por otro lado, los hospitales no se libran de los efectos de los recortes en el suministro eléctrico, y no son raros los cortes de luz, que obligan a enlentecer la actividad quirúrgica. 


\section{Resultados}

El número total de misiones efectuadas fue de doce, a falta al menos de una más, que ya está convocada para efectuarse en el próximo mes de marzo de 2020. En cada una de ellas se realizaron un total de 100 a 120 pacientes en consultas externas, de las cuales entre 20 a 30 pacientes se les hizo al menos una consulta de seguimiento. Esta ratio aumentó lógicamente en las últimas misiones. El número total de intervenciones quirúrgicas fue de 220 en total, de ellas cerca de 20 de alta complejidad, que demandaron cirugías con una duración igual o superior a dos horas.

Por razones de seguridad, en el artículo no presento la totalidad de los datos clasificados por misión, ni por fechas. Los nombres de los pacientes se han desagregado también de las bases de datos. En las tablas se nombra la patología registrada pero no en orden temporal, se nominan las tablas por letras (Tablas II, III, IV, V, VI, VII).

Tabla II Lista de patología misión A. Elaboración propia a partir del informe.

\begin{tabular}{|l|}
\hline Right DDH \\
\hline CP for Tenotomy \\
\hline CP Adductor Tightness \\
\hline Left DDH \\
\hline CP DDH \\
\hline Left DDH \\
\hline Left DDH \\
\hline talipes equinovarus (TEV) bilateral \\
\hline Steppage gait \\
\hline Recurrent Haglund Rt \\
\hline CP Tenotomy \\
\hline Congenital Knee dislocation \\
\hline Post op Right DDH \\
\hline Right LL deformity \\
\hline Gun Shot Wound Rt Hand Finger \\
\hline Epiphyseal Cyst \\
\hline Bone Cyst \\
\hline Genu valgus \\
\hline Left Leg rotational deformity \\
\hline Leg length discrepancy \\
\hline Right flat foot \\
\hline Left tibial Osgood Schlatter \\
\hline
\end{tabular}


Tabla III Diagnóstico, codificación y cirugía efectuada, misión. B. Elaboración propia a partir del informe

\begin{tabular}{|c|c|c|}
\hline Bil Ankle Equinus & Q66.0 & $\begin{array}{l}\text { Open Achilis Lenthening + } \\
\text { Dorsal Capsulorrhaphy }\end{array}$ \\
\hline Bil Madelung Deformity & Q68.1 & Lt Darrach Surgery \\
\hline $\begin{array}{l}\text { Left Thumb Congenital Trigger } \\
\text { Finger }\end{array}$ & Q66.0 & Release \\
\hline Rt Femur Head Fracture & S72.05 & Rt DHS \\
\hline Bil genu Valgus & M21.06 & Correction \\
\hline Bilateral Polydactyly & Q69.9 & Correction \\
\hline Rt foot hallux valgus & M20.1 & Osteotomy \\
\hline Rt DDH & Q65 & Open Salter Osteotomy \\
\hline Post Op for Genu Varus & M21.16 & Release Soft Tissue \\
\hline $\mathrm{DDH}$ & Q65 & Closed Reduction \\
\hline Polydactyly & Q69.9 & Amputation \\
\hline Lt tibial Osgood Schlatter & M92.42 & Excision \\
\hline Bilateral AT Equinus & M67.0 & Release and cast \\
\hline Rt CTS + Trigger Finger & M21.94 & Release \\
\hline Lt Flat Foot & Q66.4 & Arthrodesis \\
\hline Post Op & 0QP834 & Removal Implant \\
\hline Plantar Fascitis & M72.2 & $\begin{array}{l}\text { Percutaneous Plantar fascia } \\
\text { Release }\end{array}$ \\
\hline Lt Thigh Open Wound & S71.109 & Debridement and Closure \\
\hline Rt DDH & Q65 & Open Salter Osteotomy \\
\hline Drop Foot & M21.37 & Post Tibialis Transfer \\
\hline Bilateral Blount's Disease & M21.9 & Correction and Cast \\
\hline femoral epiphysial bridge & M21.9 & Excision \\
\hline Lt Medial Meniscus Injury & M17.1 & Arthroscopy \\
\hline $\begin{array}{l}\mathrm{CP}+\text { Bilateral Adductor and } \\
\text { Hamstrings Shortening }\end{array}$ & G80.4 & Tenotomy and Cast \\
\hline
\end{tabular}

Tabla IV Resumen de actividad misión C. Elaboración propia a partir del informe

\begin{tabular}{l} 
A total of 91 cases were screened, and 35 surgeries performed for 24 patients (Refer to previous \\
schedules for details) \\
\hline Pediatric population reached $82 \%$ of total screened patients and $79 \%$ of patients operated on \\
\hline Gender equality was attained within the limits of patients in need. \\
No scheduled surgeries were cancelled \\
Joint effort between expat and local teams was observed through all steps of screening, \\
properative assessment, operative procedures and postoperative follow up. \\
$\begin{array}{l}\text { Nasser Hospital's administration was made aware of all preparations, surgeries and needs } \\
\text { daily through the directors' secretary office. }\end{array}$
\end{tabular}


Tabla V Diagnóstico y tratamiento quirúrgico efectuados misión D. Elaboración propia a partir del informe Bil AT Equinus Open Achilis Lenthening + Dorsal Capsulorraphy

Bil Madelung Deformity Lt Darrach Surgery

Lt Thumb Congenital Trigger Finger Release

Bil genu Valgus Correction

Bilateral Polydactyly Correction

Rt foot hallux valgus Osteotomy

Bilateral Blount's Disease Osteotomy

femoral epiphysial bridge Excision

Lt Medial Meniscus Injury Arthroscopy

CPBilateral

Tabla VI Patología, codificación, tratamiento efectuado en la misión E. Elaboración propia a partir del informe

\begin{tabular}{|c|c|c|}
\hline Lt Tibial Bone Exostosis & Q78.6 & Excision \\
\hline Lt Elbow Varus Deformity & M21.12 & $\begin{array}{l}\text { Valgus Osteotomy of Distal Lt } \\
\text { Humerus }\end{array}$ \\
\hline TEV & Q66.0 & \\
\hline TEV & Q66.0 & \\
\hline $\begin{array}{l}\text { Healing \# after congenital } \\
\text { deformity }\end{array}$ & 0QP834 & Rush nail removal \\
\hline Cavovarus & M21.17 & Osteotomy \\
\hline Lt foot Equinus & Q66.0 & ETA + Capsulotomy \\
\hline TEV residual & Q66.0 & \\
\hline Left knee exostosis & Q78.6 & Excision \\
\hline Bilateral Hallux Valgus & M20.1 & Osteotomy \\
\hline $\begin{array}{l}\text { Posttraumatic Rt 2nd Toe } \\
\text { Deformity }\end{array}$ & M20.4 & \\
\hline Bilateral wrist deformity & Q68.1 & \\
\hline Lt TEV & Q66.0 & \\
\hline Genu Valgus & M21.06 & \\
\hline Hallux Valgus & M20.1 & \\
\hline Bilateral feet polydactylia & Q69.9 & \\
\hline Rt DDH & Q65 & \\
\hline Bil DDH & Q65 & $\begin{array}{l}\text { Pelvic Osteotomy } \\
\text { Capsulorraphy }\end{array}$ \\
\hline Lt DDH & Q65 & Capsulorraphy \\
\hline Lt DDH & Q65 & Capsulorraphy \\
\hline $\begin{array}{l}\text { Congenital both knee } \\
\text { deformity }\end{array}$ & Q68.2 & for correction \\
\hline $\begin{array}{l}\text { Rt tight Achilles tendon / } \\
\text { poliomyelitis }\end{array}$ & M67.0 & \\
\hline Old failed Lt DDH & Q65 & \\
\hline High Lt Tarsal metatarsal arch & Q66.9 & \\
\hline Bilateral genu varus & M21.16 & \\
\hline Rt genu valgus & M21.06 & \\
\hline
\end{tabular}




\begin{tabular}{|c|c|c|}
\hline Bilateral calcaneovalgus & Q66.4 & \\
\hline Penetrated DHS & T84.129 & DHS Removal \\
\hline Congenital left foot intoeing & Q66.9 & \\
\hline Bilateral genu varus & M21.16 & \\
\hline Left genu valgus & M21.06 & \\
\hline left DDH & Q65 & \\
\hline TEV & Q66.0 & \\
\hline $\mathrm{DDH}$ & Q65 & K-wire removal \\
\hline Right DDH & Q65 & \\
\hline Bilateral genu varus & M21.16 & \\
\hline $\mathrm{DDH}$ & Q65 & \\
\hline Rt TEV & Q66.0 & \\
\hline Bilateral TEV & Q66.0 & \\
\hline $\begin{array}{l}\text { Right Middle finger deformity - } \\
\text { congenital trigger finger }\end{array}$ & Q68.1 & \\
\hline Left femur anti version & Q68.3 & Lt femur osteotomy \\
\hline Left DDH & Q65 & $\begin{array}{l}\text { Open reduction } \quad+ \\
\text { capsulorraphy }\end{array}$ \\
\hline Right femur Cox varus & M21.15 & Rt femur osteotomy \\
\hline Left tibial Osgood Schlatter & M92.42 & Excision \\
\hline $\begin{array}{lll}\text { Left distal } & \text { radius } & \text { dorsal } \\
\text { angulation - } & \text { positive } & \text { ulnar } \\
\text { variance } & & \\
\end{array}$ & M21.239 & correction osteotomy \\
\hline Genu varus & M21.16 & \\
\hline Left foot Hallux valgus & M20.1 & \\
\hline $\begin{array}{l}\text { Left proximal humerus } \\
\text { exostosis }\end{array}$ & Q78.6 & \\
\hline Bilateral feet polydactyly & Q69.9 & \\
\hline Hemangioma of Rt fifth finger & D18.09 & \\
\hline Rt foot hallux valgus & M20.1 & \\
\hline Bilateral DDH & Q65 & \\
\hline Bone exostosis & Q78.6 & \\
\hline Left knee bowing - operated & Q68.2 & removal of staples \\
\hline Ganglion & M67.4 & Excision \\
\hline TEV residual & Q66.0 & \\
\hline Rt humerus \# - angulated & M21.92 & \\
\hline Posttraumatic finger Deformity & M21.94 & \\
\hline Bilateral Polydactyly & Q69.9 & \\
\hline $\mathrm{CP}$ - tight tendons & M67.0 & \\
\hline $\begin{array}{l}\text { Congenital Bilateral foot } \\
\text { deformity }\end{array}$ & Q66.0 & \\
\hline Slipped $\quad$ Capital $\quad$ Femoral & M93.003 & \\
\hline
\end{tabular}




\begin{tabular}{|c|c|c|}
\hline Epiphysis & & \\
\hline Bone exostosis & Q78.6 & Excision \\
\hline Unspecified bowing of legs & Q68.2 & \\
\hline Osteochondroma & M92.9 & Excision \\
\hline TEV residual & Q66.0 & \\
\hline $\mathrm{CP}$ - tight tendo-achillis & M67.0 & \\
\hline $\begin{array}{l}\text { Calcific Tendinitis - Plantar } \\
\text { Fasciitis }\end{array}$ & M72.2 & \\
\hline $\begin{array}{l}\text { Developmental Dysplasia of } \\
\text { the left hip (DDH) }\end{array}$ & Q65 & \\
\hline $\begin{array}{l}\text { talipes equinovarus (TEV) joint } \\
\text { bilateral fracture }\end{array}$ & Q66.0 & \\
\hline $\begin{array}{l}\text { femoral deformity genu } \\
\text { valgum }\end{array}$ & M21.06 & $\mathrm{X}$ ray $+\mathrm{MRI}$ \\
\hline $\begin{array}{l}\text { knee deformity after tibial } \\
\text { fracture }\end{array}$ & M21.96 & MRI \\
\hline $\begin{array}{l}\mathrm{CP} \text {, Both hip and knee } \\
\text { contractures } \\
\text { osteotomy femoral }\end{array}$ & M67.0 & \\
\hline Lower Limp Deformity & M21.969 & Amputation \\
\hline $\begin{array}{l}\text { developmental dysplasia of the } \\
\text { hip (DDH) }\end{array}$ & Q65 & salter and open reduction \\
\hline 2nd finger tendon tear & S66 & \\
\hline genu valgus & M21.06 & \\
\hline Posttraumatic foot deformity & M21.6 & Amputation \\
\hline $\begin{array}{l}\text { talipes equinovarus (TEV) } \\
\text { bilateral }\end{array}$ & Q66.0 & $\begin{array}{l}\text { Ilizarov correction unilateral } \\
\text { left + transference TA right }\end{array}$ \\
\hline $\mathrm{CP}$, bilateral equinovarus & Q66.0 & \\
\hline Left congenital hip luxation & Q65 & $\begin{array}{l}\text { open reduction salter } \\
\text { osteotomy }\end{array}$ \\
\hline flat foot, paresis of tendon knee & M21.40 & Follow up \\
\hline Left medial meniscus tear & M23.2 & Arthroscopy \\
\hline genu valgum left knee & M21.06 & Follow up \\
\hline Bilateral coxa vara & Q65.82 & delayed surgery \\
\hline left knee villonodular synovitis & M12.20 & arthroscopic release \\
\hline post traumatic cubitus varus & M21.12 & delayed surgery \\
\hline bilateral intoeing & Q66.9 & $\begin{array}{l}\text { corrective osteotomy and } \\
\text { external fixator }\end{array}$ \\
\hline $\begin{array}{l}\text { right foot palsy } 2 \text { ry spina bifida } \\
\text { foot peroneus luxation and } \\
\text { varus talus }\end{array}$ & Q66.9 & $\begin{array}{l}\text { calcaneal osteotomy and } \\
\text { pronouns reduction }\end{array}$ \\
\hline recurrent skewfoot & Q66.9 & open reduction capsule osteotomy \\
\hline
\end{tabular}


Tabla VII Pacientes intervenidos misión F. Elaboración propia a partir del informe

Female $3 \mathrm{~m}$. developmental dysplasia of the hip (DDH)

Male 4 y. talipes equinovarus

Male 8 y. Luxation osteotomy femoral

Female 3 y. proximal cubitus

Male 2 y. tibia varus

Male 4 y. cavus varus foot

Female 3 y. hip pain

Female 1 y. posterior tibial

Female 6 y. bilateral camptodactilia

Male Rt. knee pain

Male Rt. knee pain

Male Lt. knee pain

Male $41 \mathrm{y}$. Right knee pain medial meniscus tear

e 23 y. Left. Knee meniscus discoide tear

Male Left anterior cruciate ligament (ACL), Lateral meniscal tears

Male Right anterior cruciate ligament (ACL)

Male Right anterior cruciate ligament (ACL), Lateral meniscal tears

Female Left anterior cruciate ligament (ACL)

Male Right medial meniscus tears

Male Left medial meniscus tears

Male Left medial meniscus tears

Male Right medial meniscus tears

Las patologías más frecuentes fueron las relacionadas con la displasia del desarrollo de cadera y las grandes deformidades del pie, especialmente, el pie zambo. En la mayoría de los casos en ambas destacamos el hecho de la edad y severidad de los mismos, y el hecho que la demora en el tratamiento se relacionó con la incapacidad del sistema de salud de dar respuesta a las listas de espera. Este efecto se observa más intenso en las primeras misiones, parece debido al hecho de una mayor cercanía con la crisis bélica del verano del año 2014, cuando los servicios de traumatología se encontraban desbordados por casos de heridos urgentes. Destacan también secuelas de niños heridos por accidentes relacionados o no con el conflicto y pacientes con secuelas neurológicas y malformaciones asociadas a síndromes congénitos, osteogénesis imperfecta etc.

La actividad en el área de consultas externas se efectuó durante una jornada entera por misión, siempre con algún colega local, aproximadamente unos 120 pacientes por misión. Además de otros pacientes consultados antes y después de las jornadas quirúrgicas. Es de resaltar el valor intrínseco que una traducción directa del árabe al castellano ha ofrecido por parte de los voluntarios traductores de MdM que participaron en el proyecto. La información a los familiares, casi siempre padres, así como sus opiniones y valoraciones sobre las discapacidades de sus hijos, el valor estético y funcional, el retraso diagnóstico, así como su relación con el sistema de salud local, fue posible obtenerlo con más facilidad de este modo. En algunos casos, especialmente en el área del Hospital Nasser se observa un nivel de percepción de la enfermedad bajo, en el caso especialmente niñas, de niveles educativos familiares bajos. El impacto de los condicionantes antropológicos, de género, culturales, religiosos es alto en el proceso de toma de decisión, aunque no siempre resultó fácil de valorar por parte del autor.

El seguimiento del postoperatorio inmediato de los pacientes se realizó directamente en la visita a la planta de hospitalización, junto al traumatólogo local responsable del seguimiento del paciente y de la continuidad asistencial. Además, en las sucesivas misiones, se abrió un espacio en las agendas de consulta externa ambulatoria para dicho seguimiento (Tabla VIII). Obviamente el número de revisiones en agenda es más notorio en las últimas misiones. 
Tabla VIII Seguimiento de casos operados durante otras misiones. Elaboración propia a partir del informe Male 26 y. medial malleolus, right fracture of neck of femur dynamic condylar screw (DCS) Female 1 y. bilateral DDH,left hip capsulorrhaphy in C MM right hip capsulorrhaphy Male 11 y. road traffic accident, fracture in left leg both bone, skin defect left medial foot closed reduction left leg and elastic nails, skin graft left foot

Female 2 y. Bilateral DDH, bilateral adductor tenotomy and hip spica cast in A MM change hip spica

Female $2 \mathrm{y}$. adduction of thumb skin plastic and release skin in B MM left thumb reconstruction and skin reléase

Male 13 y. left 2nd 3rd 4th metatarsal bone fracture close reduction and wires fixation

Male 14 y. falling down left forearm fracture both bone internal fixation with elastic nail

Male 23 y. cubital tunnel syndrome, release left in A MM right ulnar nerve transposition

Male 12 y. epiphysiodesis femoral genu valgus, hemiepiphysiodesis in B MM correction steps

Female 10 y. severe tibial torsion supramalleolar osteotomy

Es relevante destacar que al menos un $10 \%$ de los pacientes atendidos ya habían sido tratados quirúrgicamente, de ellos casi la mitad, lo habían hecho en Egipto, en los momentos en el que el tránsito de pacientes era posible. Es importante reseñar la multifocalidad, o bilateralidad de muchas de las lesiones de los niños. El carácter evolutivo de la patología determina que sean precisas más de una intervención. En otros casos las intervenciones fueron realizadas por distintos equipos quirúrgicos de distintas organizaciones humanitarias en Gaza. Es una constante pues la multiplicidad de cirujanos que atendieron a los pacientes, con seguimientos en algunos casos muy cortos.

El número de intervenciones suspendidas fue de 15, en 6 ocasiones por razones relacionadas con el estado de salud de los niños, por patología médica no relacionada, habitual en niños con síndromes malformativos múltiples, parálisis cerebral infantil (PCI o CP). Estos niños no tienen en muchos casos un tratamiento crónico de soporte adecuado para su estado basal. En el resto de los casos la suspensión fue debida a problemas técnicos relacionados con no disponer de material o instrumental adecuado para la intervención, especialmente placas de osteotomía adecuadas al hueso infantil, instrumental de precisión para microcirugía, sistemas de corte óseo de precisión. En otras el problema es la falta de stock suficiente de materiales implantables. El índice de ocupación del quirófano fue alto, cercano al 60\%, aunque en unas siete intervenciones la duración de la cirugía se prolongó una media de cinco minutos, hasta diez minutos en un caso, debido a cortes en el suministro eléctrico. Dichos cortes suponen la entrada de los generadores que nutren a los aparatos de soporte vital, pero los sistemas de imagen, radioscopia, tienen que volver a ser reconectados en muchas ocasiones son las consiguientes demoras.

La actividad formativa y de capacitación fue realizada en varios modos. A destacar la participación de los traumatólogos locales durante la actividad tanto en las consultas externas (Figura 4a), así como durante las sesiones quirúrgica (Figura 4b). Aproximadamente el $45 \%$ de las intervenciones el cirujano principal fue el traumatólogo local, con la ayuda de algún traumatólogo del EQE. La actividad training in work se complementó con la participación de los traumatólogos del EQE en la visita a la planta de hospitalización, junto con los colegas locales, así como a las sesiones clínicas propias del servicio de traumatología diarias (Figura 5). La actividad específica de formación de la misión de febrero de 2015 contó con la participación de más de 50 profesionales del ámbito de la Ortopedia Infantil, muchos de los cuales participaron después en las sucesivas misiones, algunos en distintos hospitales de la Franja, pues a lo largo de los años cambiaron su destino en otros hospitales. Parte de la actividad formativa se continuó después on line, debido al contacto mantenido con los colegas por parte de los miembros el EQE. 


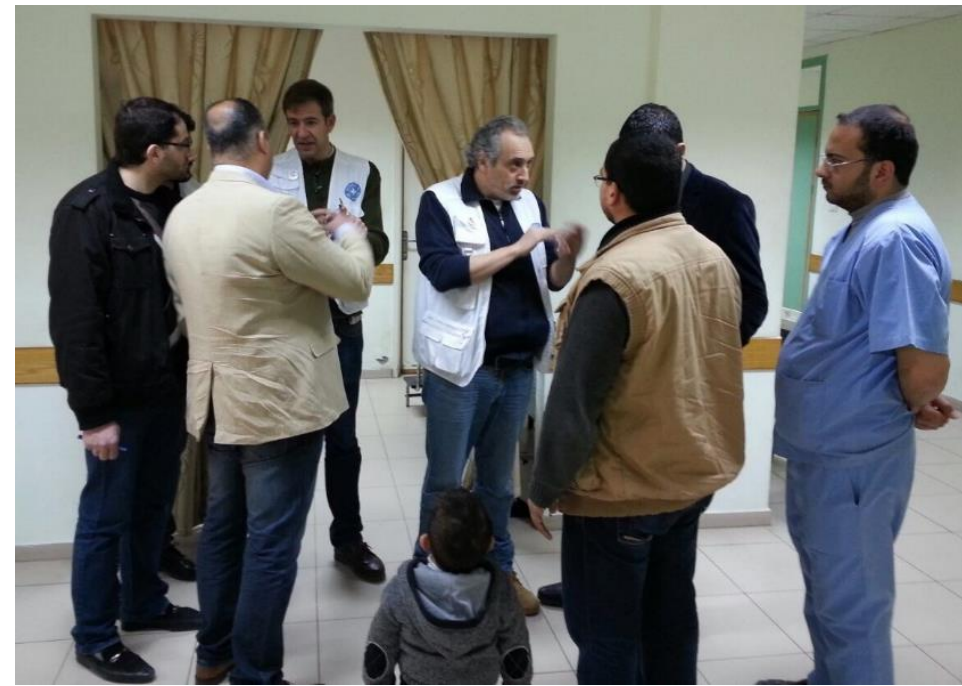

(a)

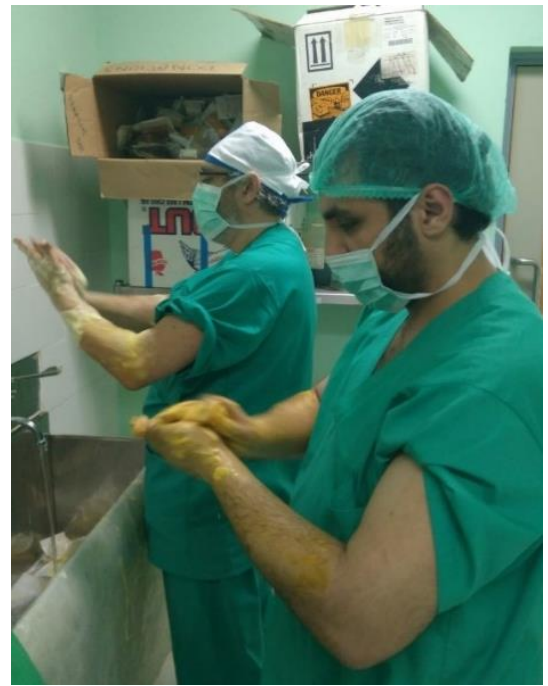

(b)

Figura 4 Participación de los cirujanos locales

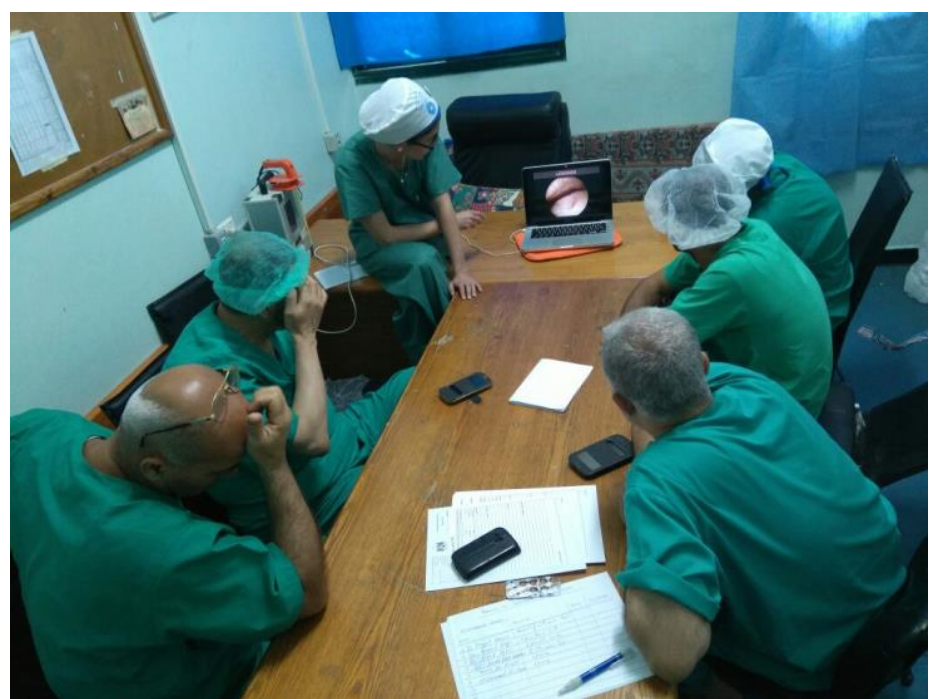

Figura 5 Sesiones diarias de traumatología con el equipo local

Tras los sucesivos informes de actividad que los miembros del EQE aportaron a la organización MdM, se han podido implementar actividades de mejora del programa. La distribución de área de preferencia de la intervención, los facultativos más implicados en la capacitación, o las listas de prioridades para la entrega y donación de material quirúrgico para el ámbito de la Traumatología Infantil, fueron ejemplo de algunos cambios.

\section{Discusión}

Los proyectos de $\mathrm{AH}$ son exigentes en cuanto a su despliegue. La implantación en el terreno de una organización como MdM [12], permitió que el MoH se fijara en dicha organización para la implementación de este programa. Es necesario que la organización disponga de una oficina permanente dentro de Gaza, así como implantación en el resto de TOP para poder establecer dichas relaciones. La sola dificultad de los permisos de paso obliga a una buena relación con las autoridades israelíes. El contexto inestable, con repetidas situaciones de recrudecimiento de hostilidades, obligan a la organización a tener presente de forma permanente y revisable la situación de seguridad. Se hace casi imposible diferenciar entre una situación permanente de dificultades de desarrollo con una crisis humanitaria [13]. Sólo una buena y exigente actividad de los equipos locales de la organización permiten poder implementar este tipo de actividades. Si nos fijamos en los resultados podemos ver 
que el número de misiones y su separación temporal condiciona mucho dicho despliegue. Las circunstancias cambiantes, y la necesidad de mejorar el impacto, determinaron pues que el programa se centrase en el Hospital de Nasser, una zona más deprimida socioeconómicamente en la Franja, y dónde la colaboración con los traumatólogos locales alcanzó una mayor aceptabilidad del proyecto. El objetivo de mejorar la calidad de la respuesta, la aceptación de las normas mínimas universales [14], y la mejora en la rendición de cuentas ante la población afectada obligan a amentar dichos niveles de exigencia en el proyecto. Este ejemplo no podía ser menos.

$\mathrm{Si}$ los proyectos de $\mathrm{AH}$ son exigentes, dentro de ellos los proyectos de EQE obligan a unos niveles de mínimos sobre capacidades de alta [7, 15]. En el Manual de Acción humanitaria de MdM [12] se establecen como recursos humanos básicos para un bloque quirúrgico el formado por un coordinador/a de intervención (preferentemente del asociativo), un médico de urgencias-traumatólogo, dos DUE, una persona de apoyo, a valorar entre auxiliar de enfermería o técnico de emergencias, un Logista un Administrador/a RRHH Completo: 1 Anestesista 1 Traumatólogo 1 Cirujano general 1 Cirujano vascular 1 Cirujano plástico Equipamiento y kits básicos por bloque quirúrgico: Shelter: Infraestructura o Hospital de campaña reducido compuesto por mínimo 4 módulos (prequirúrgico, quirúrgico, postquirúrgico, farmacia) con suministro de luz y electricidad ininterrumpida y agua potabilizada/higienizada. En cualquiera de los casos, será prescindible habilitar espacios específicos para: - Zona Pre Quirúrgica-Radiología - Quirófano - Zona Post Operatorio/UCI - Zona de esterilización - Farmacia - Banco de sangre - Tratamiento de desechos.

En este proyecto estas infraestructuras son las propias de los hospitales del sistema público de salud palestino locales. Se trata de un proyecto de fortalecimiento que no de sustitución del mismo. En este caso las exigencias cambian ya que el tipo de actividad se ciñe más a una parte específica de la patología. Si bien el proyecto se va a desarrollar en estructuras físicas útiles, en hospitales con capacidad operativa, aquí es la demanda de la complejidad de la patología la que obliga a una mayor especialización de los miembros del equipo. La necesidad de aportar valor supone que la subespecialización obliga a una selección y por tanto a una menor disponibilidad de profesionales. La captación de los mismos supuso un arduo trabajo, pues el contexto de Gaza a muchas personas hecho para atrás, y por tanto completar los equipos no siempre fue fácil. En todo caso, el hecho de que la actividad se realizase junto a los traumatólogos locales permitió poner en valor lo cualitativo además de lo cuantitativo, poder seleccionar los casos con cirugía más complejas, en todas las misiones los resultados nos indican este hecho. Poder considerar pues el impacto de los resultados cuantitativos, que puede que se resientan en cifras, si lo comparamos son necesidades percibidas, pacientes en lista de espera para una intervención. Es importante resaltar ese indicador de actividad y el resultado de las intervenciones complejas, por ejemplo, osteotomías pélvicas para la DDH, o reconstrucciones de TEV severos recidivados.

La patología en el ámbito de la Ortopedia Infantil es muy dependiente de las capacidades de atención del sistema de salud. Este es un hecho constatable en todos los contextos, aun en sistemas de salud de alto nivel, esa máxima se cumple. En muchas ocasiones la pluripatología de los pacientes afectados, o la saturación de los recursos sanitarios hace que este grupo de enfermedades pasen a un segundo plano en las prioridades asistenciales. Si le añadimos a ello el peculiar contexto de Gaza [16], podemos detectar como la lista de espera de los casos se paraliza en cuanto a nuevos casos que entran durante las fases de crisis, por tanta llegada de casos de urgencias a los hospitales, así como de salida de pacientes de dicha lista por ser intervenidos, se reduce significativamente. Después, en las fases de menor demanda de urgencias vuelve a incrementarse el número de casos que entran en la lista, y ahora los procesos están más evolucionados en el tiempo, y por tanto con una solución quirúrgica más compleja. Además, la patología en la ortopedia infantil obliga a dar seguimiento a los pacientes durante varios años, en general toda la etapa de crecimiento para poder hacer una evaluación adecuada de los procedimientos y tratamientos. Aunque el proyecto tiene vocación de dar una mínima respuesta a esta necesidad, la enorme debilidad del sistema de salud no permite poder responder con máxima eficacia. Podemos ver en los resultados de actividad que el número de pacientes atendidos dentro del proyecto respecto a número de pacientes afectados es una ratio 
inalcanzable. Sólo un refuerzo estructural de capacidades, por ejemplo, al menos una Unidad de Referencia en la Franja, como se establece en los objetivos a medio largo plazo dentro del Hospital Nasser, podría tener impacto cuantitativo. El nivel de demanda asistencial pone a prueba al sistema en su conjunto, desde pediatría de primaria, hasta servicios de neurología, como el caso de pacientes con parálisis cerebral infantil (PCI) o rehabilitación se ven involucrados en la atención integral de los pacientes. Los métodos de screening poblacional a patologías como por ejemplo la displasia del desarrollo de cadera necesitan unas guías clínicas precisas para la derivación. Incluso en la fase de tratamiento, es necesario disponer también de una buena estructura con capacidades para adaptar aparatos ortopédicos adecuados y de calidad. Todos estos ejemplos suponen una condición para una buena atención en Ortopedia Infantil. Un nivel de exigencia difícil de conseguir en la Franja.

Al hacer un análisis de los resultados de las indicaciones quirúrgicas y opciones de tratamiento en las consultas externas hemos podido comprobar como la demanda de atención tiene unos importantes sesgos dependientes del contexto socioeconómico [17, 18]. Este hecho fue un factor determinante, entre otros para enfocar el proyecto en el área de Khan Younis, más deprimida socialmente. Entre las observaciones detectadas, en gran parte gracias a la comunicación directa, a través de nuestros traductores, con los padres, destacan ejemplos como la demanda estética de las malformaciones de los tobillos en el caso de las niñas, u otros casos como padres que solicitaban una solución quirúrgica a un problema determinado, por no disponer de dinero para comprar algún aparato ortopédico, teniendo en cuenta que la cirugía tendría menos costes. Hemos visto pues como algunos elementos culturales, económicos, de género, religiosos, sociales se interrelacionan con la percepción de salud y enfermedad, tolerancia a la incapacidad e incluso imagen corporal. Muy significativo también es la prevalencia familiar de algunas enfermedades, como algunas familias con varios hermanos con osteogénesis imperfecta. El consejo genético adquiere unos importantes matices en cuanto al contexto, y precisa de una importante adaptación y enfoque antropológico.

A pesar de que, en comparación con otras partes de la medicina, e incluso de la traumatología, el material en su mayoría de uso habitual en Cirugía Ortopédica Infantil es menos demandante en complejidad y precio. Sin embargo, hay algunos casos concretos, como las placas para osteotomías, clavos de crecimiento óseo, sistemas de fijación externa específicos para la infancia, material de microcirugía, técnicas de cirugía mínimamente invasivas, que no están disponibles en la zona. Esto supone que las opciones quirúrgicas se reduzcan, en ocasiones hace que la técnica no sea posible o se haga con mayor dificultad. En nuestras misiones en varias ocasiones hemos tenido que posponer o suspender intervenciones por este hecho. Además, tenemos que considerar que el mantenimiento del instrumental quirúrgico también tiene importantes handicap, como la reposición de implantes quirúrgicos en número suficiente, la velocidad para la esterilización con garantías del material y la calidad del mismo. Hasta en varias intervenciones se han prolongado los tiempos de la operación por no disponer por ejemplo de algún material, o debido a los cortes en el suministro eléctrico, ya que, aunque el sistema autónomo de generadores mantiene los sistemas de soporte vital activos, se puede afectar al funcionamiento de los aparatos de radioscopia necesarios para múltiples intervenciones en cirugía ortopédica. En el contexto del bloqueo al que está sometida la zona, el transporte de materiales e instrumentales quirúrgicos de calidad para intervenciones complejas se convierte en un auténtico desafío.

Gran parte del material e instrumental de los centros hospitalarios es fruto de las donaciones de agencias internacionales y ONGs, no siempre los suministros se pueden garantizar y adaptar adecuadamente a las necesidades, pues la lentitud de los permisos de paso de estos no lo permite [20]. En el ámbito del proyecto de MdM podemos resaltar la posibilidad que tuvo la organización de donar y aportar la organización de instrumental e implantes de enclavamiento endomedular con clavos elásticos tipo Nancy, útiles para el uso en traumatología infantil.

El contexto del bloqueo en la Franja de Gaza no sólo afecta a los materiales sino también a las personas. Aunque existe sistemas para mantener un corredor humanitario para el paso de pacientes, los permisos son lentos, las exigencias de acompañamiento altas, muy trascendente para el caso de niños y niñas que apenas pueden ser acompañados en el tránsito [21]. Además, la patología relacionada con la Ortopedia Infantil no está considerada trascendente para ser trasladada, respecto 
a otro tipo de patologías y muchos permisos no son considerados. Parece que la salud se convierte en un sector residual en el ámbito de la cooperación internacional [22], por ejemplo, durante no pocos años ha dejado de ser prioritario para Palestina por parte de la AOD de España [23]. Eso supone dificultades para conseguir financiar los proyectos [24].

Los datos del último informe sobre "La salud en la cooperación al desarrollo y la acción humanitaria 2017" [25], de la Fundación para la Cooperación Internacional de la Organización Médica Colegial (FCOMCI), una publicación on line que recopila las noticias más destacadas de este ámbito, la actividad de la Fundación y la labor humanitaria de los Colegios de Médicos advierten que la cooperación española al desarrollo en materia de salud "se ha desplomado" y se ha convertido en un "sector residual" dentro de la acción humanitaria en España. La salida de los pacientes para ser tratados se produce de forma muy desigual, eso supone que un mismo paciente puede ser tratado en puntos muy dispares, hospitales de Egipto, cuando el paso sur por Rafah está abierto, Jerusalén Este, cuando Israel da permiso, dentro de la Franja, cuando es atendido por algún proyecto de una ONG o bien por los traumatólogos locales. Esta múltiple asistencia de un mismo paciente va en contra de una atención más integral de pacientes complejos, en patologías que evolucionan durante años.

Este bloqueo para el tránsito de personas incluye y afecta también a los profesionales. Nuestros compañeros traumatólogos no pueden salir, o tienen grandes dificultades con los permisos de salida [26]. La posibilidad de compartir cursos de formación, congresos, talleres de capacitación en nuevas técnicas quirúrgicas de los colegas locales se reduce de forma importante. El hecho de que el proyecto se desarrolla dentro de los hospitales público, con sus recursos y junto los colegas locales, que seleccionan, y hacen seguimiento postoperatorio a los pacientes, permite implementar en el mismo actividades de formación in working. El trabajo tanto en consulta externa como en el quirófano se realiza junto con los traumatólogos locales. La valoración de esta parte del proyecto por parte de los profesionales es muy alta y la búsqueda de nuevas implementaciones en el mismo, como formación y acompañamiento on line, tramitación o facilitación de salidas formativas, adquieren importancia.

Es importante reseñar que el inicio del proyecto es respuesta a una solicitud del Ministerio de Salud Palestino $(\mathrm{MoH})$, gracias a la buena relación con MdM, en relación a proyectos previos. En una primera fase misional exploratoria, se realizó una actividad grupal, en formato congreso, en febrero de 2015, donde acudieron personas de distintos sectores relacionados con la Ortopedia Infantil. En dicha reunión se puso de manifiesto la necesidad percibida por los profesionales de implementar medidas en la materia. Incluso de aportaron datos al respecto de una mayor incidencia de malformaciones congénitas en la zona en diversas etapas temporales, coincidentes con momentos de escalada militar y bombardeos. Muchas de estas afirmaciones no pudieron ser contrastadas con datos. Se puso en evidencia la no disponibilidad de datos epidemiológicos consistentes en Ortopedia Infantil sobre los que poder sacar conclusiones [27-29]. En la implementación de algún proyecto ad hoc se hace complejo medir datos de impacto cualitativo o cuantitativo, tanto actual como de salud futura.

\section{Conclusiones}

Los proyectos de $\mathrm{AH}$ son exigentes. Es necesario una estructura organizativa con capacidades estructurales altas para implementarlos. Especialmente importante es esto en un contexto cambiante como Gaza, donde la sola burocracia para poder trasladar personas con seguridad y materiales con calidad consume una alta cantidad de recursos.

El EQE es un método con impacto en el ámbito de la AH. Las capacidades de los miembros del equipo para la resolución de patologías complejas hacen que el impacto cualitativo de la intervención sea alto y se puedan resolver o paliar muchas patologías que de otro modo no se curarían.

La ortopedia infantil da información relevante sobre la situación de capacidades de respuesta del sistema sanitario. Los indicadores de resultado de este ámbito son útiles para saber cómo de bien 
funciona un sistema sanitario, pues muchas de las consecuencias de las deficiencias se arrastrarán por años y limitarán la salud y la calidad de vida de muchas personas.

La ortopedia infantil da información relevante sobre el contexto socioeconómico, político, religioso. Un análisis de género de los datos y un enfoque antropológico de los mismos darían una información muy útil y relevante para el conocimiento del medio.

El bloqueo de materiales e instrumentales afecta a las capacidades operativas de los profesionales locales y de los miembros EQE. Esto tiene consecuencias finales sobre el estado de salud de las personas.

El bloqueo de personas afecta al estado de salud. Se dificulta el tránsito de pacientes para ser tratados fuera de Gaza en mejores condiciones. Pierde relevancia la salud en general y no se le da importancia a la patología del ámbito de la ortopedia infantil en particular, por comparativa a otros procesos más sensibles, o considerados más importantes y eso afecta a la calidad de vida niños y niñas y por ende la salud futura de varias generaciones.

El bloqueo de personas afecta a la movilidad de los profesionales locales, y les ocasiona no disponer de una formación técnica actualizada lo que disminuye sus capacidades. La formación in working que puede proporcionar un EQE es considerada de alto valor por los profesionales locales.

No disponer de datos epidemiológicos no permite hacer planes asistenciales adecuados a las necesidades reales. El contexto inestable afecta al sistema de salud, y las listas de espera de Ortopedia Infantil no se mueven durante las fases de recrudecimiento del conflicto, durante el que los servicios de Traumatología se centran en la atención a urgencias. La multiplicidad de actores en la actividad asistencial no ayuda a resolver la situación, pues no siempre coinciden temporalmente con las necesidades.

Agradecimientos: Este artículo sería imposible sin la participación de todas aquellas personas voluntarias que formaron parte de los distintos EQE: 12 traumatólogos y traumatólogas, 8 anestesistas, una enfermera de quirófano, que acudió en todas las misiones. El personal local de MdM en la Franja: la coordinadora de misión en Gaza, dos enfermeros y un médico de enlace con el personal local y las autoridades sanitarias. Las tres personas coordinadoras de país durante el período de actividad del proyecto. Los conductores de MdM dentro de la Franja que nos trasladaron a los hospitales con máxima seguridad. Las dos personas voluntarias dentro de la Franja que nos sirvieron de traductoras. La ayuda en algunas misiones de dos personas de voluntariado y comunicación. En suma, el trabajo conjunto de muchísimas personas, sin olvidarme de mis compañeros de los sistemas de salud gazatíes, mis colegas traumatólogos, personal de enfermería, anestesia, pediatras, etc. Es debido a razones de seguridad que no resulta adecuado dar sus nombres, pero sí un agradecimiento expreso a todas ellas.

Conflicto de Intereses: El autor no declara conflicto de intereses. El autor no identifica ni declara ninguna circunstancia personal o interés que pueda percibirse como que influye indebidamente en la representación o interpretación de resultados de la investigación divulgada. El autor cuenta con los datos recogidos por la organización MdM y con el consentimiento expreso de la misma. La organización MdM no tiene ningún papel en el diseño del estudio; en el análisis o interpretación de los datos; en la escritura del manuscrito y en la decisión de publicar los resultados. Son responsabilidad el autor las opiniones y conclusiones del artículo.

\author{
Abreviaturas \\ Las siguientes abreviaturas son usadas en este manuscrito: \\ TOP: Territorios Ocupados Palestinos \\ EQE: Equipos quirúrgicos especializados \\ SST: Specialized Surgical Team \\ UNRWA: Agencia Naciones Unidas para Refugiados Palestinos en Oriente Próximo \\ MdM: Médicos del Mundo \\ ECHO: Agencia Europea para Acción Humanitaria \\ OCHA: Agencia de Naciones Unidas para Acción Humanitaria \\ MoH: Ministerio Palestino de Salud \\ DDH: Dysplasia devolvement hip, Displasia desarrollo de cadera
}


TEV: Talipes equino varo, Clubfoot o pie zambo

AH: Acción Humanitaria

MM: Misión médica

DCS: Dynamic condylar screw, placa condilar dinámico

WHO: World Health Organization

OMS: Organización Mundial de la Salud

ACL: Anterior Cruciate Ligament, Ligamento cruzado anterior

TA: Tendón Tibial Anterior

CP: Cerebral Palsy

PCI: Parálisis Cerebral Infantil

CRE: Cruz Roja Española

ONG: Organizaciones No Gubernamentales

CIRCR: Comité Internacional de la Cruz Roja

TFM: Trabajo Fin de Máster

\section{Referencias Bibliográficas}

1. More, Anne Le. "International Assistance to the Palestinians after Oslo: Political Guilt, Wasted Money", Routledge Studies on the Arab-Israeli Conflict. Routledge, 2008.

2. Mcneely, Clea A., et al., “Long-Term Health Consequences of Movement Restrictions for Palestinians, 1987-2011", American Journal of Public Health, vol. 108, no. 1, 2018, pp. 77-83., doi:10.2105/ ajph.2017.304043.

3. Marcos Tamaríz, Louise Ashwell. “The labyrinths to health in Gaza”. Dic 2019.

4. "OCHA, Gaza Strip: Early warning indicators"- September 2019. 21 October 2019. https://www.ochaopt.org/content/gaza-strip-earlywarning-indicators-september-2019

5. Gerald Rockenschaub, Head of oPt, Mahmoud Daher, Head of Gaza sub-office, Sara Halimah, Technical Officer Health Cluster \& Emergencies, Abdelnaser Soboh, National Officer Health Cluster, “WHO Special Situation Report Gaza, occupied Palestinian territory". Agosto 2017.

6. World Health Organization - Health Cluster. "Situation Report oPt Gaza”, 1-31 March 2019. http://www.emro.who.int/images/stories/palestine/documents/WHO_Special_Situation_report_SitRep_ March_2019.pdf?ua=1

7. Beatriz Martínez. Junio 2019. “Análisis crítico del Equipo Técnico Español de Ayuda y Respuesta a Emergencias (START) dentro del marco de la iniciativa de Equipos Médicos de Emergencias (EMT) de la Organización Mundial de la Salud". pp 9-10. TFM Máster Acción Humanitaria y Salud Universidad Alcalá de Henares.

8. WHO, "Emergency Trauma response to the Gaza Mass Demonstrations 2018-2019 - A one-year review of trauma data and the humanitarian consequences", May 2019. http://www.emro.who. int/images/stories/palestine/documents/who_emergency_trauma_ response_to_gaza_demonstrations_2018_2019.pdf?ua=1\&ua=1

9. UN OCHA, “Approaching the first anniversary of the 'Great March of Return' protests in Gaza”, The Monthly Humanitarian Bulletin: OPT - March 2019. Published on 27 March 2019.

https://www.ochaopt.org/content/approaching-first-anniversary-great-march-return-protests-gaza

10. WHO, "Emergency Trauma response to the Gaza Mass Demonstrations 2018-2019 - A one-year review of trauma data and the humanitarian consequences", May 2019.

http://www.emro.who.int/images/stories/palestine/documents/who_emergency_trauma_ response_to_gaza_demonstrations_2018_2019.pdf?ua $=1 \& u a=1$.

11. UN News, “Hundreds of wounded Gaza protesters risk limb amputation without immediate help, warns top UN official", 8 May 2019. https://news.un.org/en/story/2019/05/1038101

12. "Manual de procedimientos y estrategia en Acción Humanitaria de Médicos del Mundo". Julio 2016.

13. Diakonia, "The Humanitarian - Development Divide: A False Dichotomy? - The International Law Framework for Humanitarian and Development Assistance in a Context of Protracted Occupation." Diakonia International Humanitarian Law Resource Centre, August 2018.

14. CICR “El Manual Esfera". "Carta Humanitaria y Normas Mínimas para la respuesta humanitaria”.

15. Fdez Palacios J. Mayo 2015 “Cirugía Plástica en Emergencias Humanitarias: 20 años de experiencia con Médicos del Mundo”. Cirugía Plástica Iberoamericana Vol. 41- nº 2. pp203-211. 
16. 2016. Doi: 10.17265/1548-6648/2016.02.006. 13 AIDA, “50 Years of Occupation: Dispossession, Deprivation and Development", October 2017.

17. WHO Palestine Office. "The humanitarian impact of the internal palestinian divide of the Gaza Strip". Junio 2017.

18. UNCTAD, “UNCTAD assistance to the Palestinian people: Developments in the Economy of the occupied Palestinian territory", September 2017.

19. Palestinian Central Bureau of Statistics, Poverty Profile in Palestine. "The PCBS defines individuals that live below the poverty line are unable to acquire the necessities of food, clothing and shelter". PCBS, 2017.

20. Van Hollen, Chris and USAID “Impact of Foreign Assistance Cuts to the West Bank and Gaza". https://www.vanhollen.senate.gov/imo/media/doc/Impact\%20of\%20Foreign \%20Assistance\%20Cuts $\% 20$ to\%20the\%20West\%20Bank\%20and\%20Gaza.pdf

21. Holmes, Oliver and Hazem Balousha "A Jerusalem hospital where Palestinian babies die alone", The Guardian, 20 June 2019. https:// www.theguardian.com/world/2019/jun/20/a-jerusalem-hospitalwhere-palestinian-babies-die-alone

22. C. Mediano, E. Tapia. MdM, Medicos Mundi, Prosalud, "La Salud en la Cooperación al Desarrollo y la Acción Humanitaria “. 2016, 2017, 2018.

23. J.P de la Iglesia y González de Peredo. "Estrategia de Acción Humanitaria de la Cooperación Española". Mayo 2019.

24. OCHA, “OPT: Humanitarian Funding Update-31 August 2019”, 2019. https://www.ochaopt.org/sites/default/files/funding_dashboard_august_2019.pdf

25. Fundación para la Cooperación Internacional de la Organización Médica Colegial (FCOMCI), "La salud en la cooperación al desarrollo y la acción humanitaria 2017",

http://www.medicosypacientes.com/articulo/la-salud-un-sector-residual-en-la-cooperacion-al-desarrolloespanola

26. Kubovich, Yaniv. “35,000 Palestinians Left Gaza in 2018; Hamas Blocking Doctors from Leaving” Haaretz, 19 May

2019.https://www.haaretz.com/middle-east-news/palestinians/.premium-35000-palestinians-left-gaza-in-2 018-hamas-blocking-doctors-fromleaving-1.7254747

27. State of Palestine Ministry of Health, “Health Annual Report Palestine 2018”. Palestinian Health Information Centre. July 2019.

28. Palestinian Central Bureau of Statistics, "Palestinian Health Accounts 2017", Ministry of Health, Ramallah, Palestine, 2019.

29. Palestinian Central Bureau of Statistics, "Quarterly national accounts variables in Palestine 2011-2019”, PCBS, 2019. http://www.pcbs.gov.pp/Portals/_Rainbow/Documents/E.QNA_Current.html 